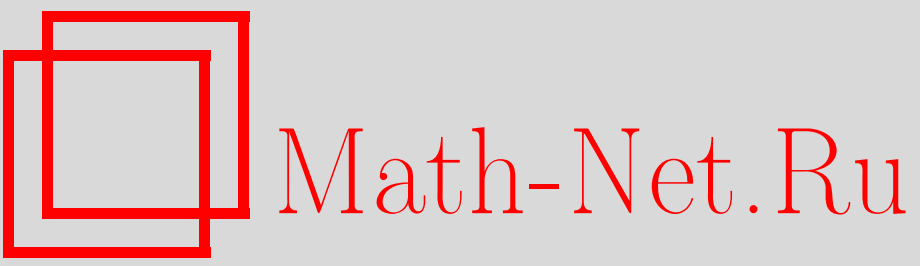

Д. В. Ионеску, Можно ли перенести понятие однородного гравитационного поля из классической механики в релятивистскую теорию гравитации?, ТМФ, 2002, том 130, номер 2, 339-352

DOI: https://doi.org/10.4213/tmf306

Использование Общероссийского математического портала Math-Net.Ru подразумевает, что вы прочитали и согласны с пользовательским соглашением

http: //www . mathnet.ru/rus/agreement

Параметры загрузки:

IP : 44.207 .124 .84

26 апреля 2023 г., 13:10:40 
ТЕОРЕТИЧЕСКАЯ

И МАТЕМАТИЧЕСКАЯ

ФИЗИКА

Том 130, № 2

февраль, 2002

(C) 2002 г.

Д. Ионеску*

\section{МОЖНО ЛИ ПЕРЕНЕСТИ ПОНЯТИЕ ОДНОРОДНОГО ГРАВИТАЦИОННОГО ПОЛЯ ИЗ КЛАССИЧЕСКОЙ МЕХАНИКИ В РЕЛЯТИВИСТСКУЮ ТЕОРИЮ ГРАВИТАЦИИ?}

\footnotetext{
Обобщение концепции однородного поля тяготения классической механики рассматривалось на основе общей теории относительности Эйнштейна Богородским. В предлагаемой статье такое обобщение ищется в случае релятивистской теории гравитации. Имеется сушественная разница между соответствующими решениями в этих двух теориях. Решение, полученное согласно релятивистской теории гравитации, не удовлетворяет принципу причинности в этой теории. Таким образом, проблема построения обобщения классического понятия однородного гравитационного поля в рамках релятивистской теории гравитации остается открытой.
}

\section{1. ВВЕДЕНИЕ}

В классической механике Ньютона однородное поле тяготения - это поле, которое в каждой точке имеет один и тот же градиент потенциала. Такое поле порождается бесконечной материальной плоскостью с постоянной поверхностной плотностью массы (см. далее раздел 3 ).

Можно ли сохранить классическое понятие однородного гравитационного поля в релятивистской теории гравитации (РТГ)? При анализе этой проблемы исходным пунктом являлась монография Богородского [1] (гл. 17), где автор рассматривает в общей теории относительности Эйнштейна (ОТО) проблему определения поля тяготения, порожденного системой масс, равномерно распределенных на плоскости. Ниже в разделе 4 описано, что именно Богородский понимает под однородным полем тяготения в ОТО. Как будет видно, его решение имеет неустранимую сингулярность, которая появляется без какого-либо физического объяснения.

Проблема однородного поля тяготения в РТГ была кратко рассмотрена в статье [2]. В разделе 5 данной работы эта проблема анализируется подробно. Решение полной сис-

* Department of Mathematics, Technical University of Civil Engineering, Bucharest, Romania. E-mail: dionescu@hidro.utcb.ro 
темы уравнений РТГ для рассматриваемой задачи отличается от решения, полученного Богородским. Построенное решение регулярно во всей области определения, но не может быть принято в качестве реального поля тяготения, имеющего физический смысл, потому что оно не удовлетворяет принципу причинности РТГ. Таким образом, проблема нахождения этого поля в РТГ остается открьтой.

В разделе 6 показано, что если все же использовать найденное решение, то окажется, что скорость некоторых свободных пробных частиц в полученном поле будет превосходить скорость света в вакууме.

\section{2. УРАВНЕНИЯ РТГ И ПРИНЦИП ПРИЧИННОСТИ В РТГ}

РТГ была построена Логуновым и его сотрудниками (см. [3], [4]) как полевая теория тяготения на основе специальной теории относительности (СТО). Пространство-время Минковского является здесь фундаментальным пространством, которое содержит все физические поля, включая гравитацию. Элемент длины в этом пространстве дается формулой

$$
d \sigma^{2}=\gamma_{m n}(x) d x^{m} d x^{n}
$$

где $x^{m}, m=1,2,3,4,-$ допустимые координаты в пространстве-времени Минковского; $\gamma_{m n}(x)$ - компоненты метрики Минковского в выбранной координатной системе.

Гравитационное поле описывается симметричным тензором второго ранга $\phi^{m n}(x)$, благодаря которому возникает эффективное риманово пространство-время. Одно из основных предположений РТГ говорит, что поведение материи в пространстве-времени Минковского с метрикой $\gamma_{m n}(x)$ под действием гравитационного поля $\phi^{m n}(x)$ эквивалентно ее поведению в эффективном римановом пространстве-времени с метрикой $g_{m n}(x)$, определенной соотношением

$$
\tilde{g}^{m n}=\sqrt{-g} g^{m n}=\sqrt{-\gamma} \gamma^{m n}+\sqrt{-\gamma} \phi^{m n}, \quad g=\operatorname{det}\left(g_{m n}\right), \quad \gamma=\operatorname{det}\left(\gamma_{m n}\right) .
$$

Такое взаимодействие гравитационного поля с материей было названо принципом геометризации РТГ.

Поведение гравитационного поля определяется следующими дифференциальными уравнениями РТГ:

$$
\begin{gathered}
R_{n}^{m}-\frac{1}{2} \delta_{n}^{m} R+\frac{m_{\mathrm{g}}^{2}}{2}\left(\delta_{n}^{m}+g^{m k} \gamma_{k n}-\frac{1}{2} \delta_{n}^{m} g^{k l} \gamma_{k l}\right)=8 \pi T_{n}^{m}, \\
D_{m} \tilde{g}^{m n}=0, \quad m, n, k, l=1,2,3,4 .
\end{gathered}
$$

Здесь $R_{n}^{m}$ - тензор Риччи, соответствующий метрике $g_{m n}, R=R_{m}^{m}$ - скалярная кривизна, $\delta_{n}^{m}$ - символ Кронекера, $m_{\mathrm{g}}$ - масса гравитона, а $T_{n}^{m}$ обозначает тензор энергии-импульса источников гравитационного поля. В уравнении $(2.4) D_{m}$ - оператор ковариантного дифференцирования по отношению к метрике $\gamma_{m n}$. Уравнения $(2.3),(2.4)$ 
являются ковариантными при произвольных координатных преобразованиях с ненулевым якобианом. В РТГ все полевые переменные зависят от универсальных пространственно-временных координат пространства Минковского. Присутствие массовых членов в уравнении (2.3) позволяет однозначно определить геометрию пространства-времени и плотность энергии-импульса гравитационного поля при отсутствии материи. Уравнение (2.4) говорит нам, что гравитационное поле обладает только спиновыми состояниями 0 и 2. В работе [4] это уравнение, определяющее состояния поляризации поля, оказывается следствием того, что источником гравитационного поля является универсальная сохраняюшаяся плотность тензора энергии-импульса всей материи, включающей гравитационное поле. Масса гравитона существенно влияет на эволюцию Вселенной и изменяет характер гравитационного коллапса.

В данной работе из-за чрезвычайной малости массы гравитона $\left(m_{\mathrm{g}} \simeq 10^{-66}\right.$ г) мы будем анализировать проблему нахождения однородного гравитационного поля в РТГ, рассматривая уравнение (2.3) без массовых членов. Во всех уравнениях ниже используются релятивистские единицы.

Уравнение (2.4) может быть записано в следуюшем виде (см. [3], приложение 1):

$$
D_{m} \tilde{g}^{m n}=\tilde{g}^{m n}{ }_{m}+\gamma_{m p}^{n} \tilde{g}^{m p}=0
$$

где $\gamma_{m p}^{n}$ - компоненты метрической связности, порожденной $\gamma_{m n}$, запятая в (2.5) обозначает производную по соответствуюшей координате.

Принцип причинности в РТГ предложен и проанализирован Логуновым в книге [4], гл. 6. Согласно этому принципу любое движение точечного пробного тела должно осуществляться в пределах причинного светового конуса пространства-времени Минковского. Согласно анализу Логунова принцип причинности будет выполняться тогда и только тогда, когда для любого изотропного вектора Минковского $u^{m}$, т.е. для любого вектора, удовлетворяющего условию

$$
\gamma_{m n} u^{m} u^{n}=0
$$

метрика эффективного риманова пространства-времени будет удовлетворять ограничению

$$
g_{m n} u^{m} u^{n} \leqslant 0 .
$$

Согласно принципу причинности в РТГ физический смысл могут иметь только те решения системы (2.3), (2.4), которые удовлетворяют этому ограничению.

Важно подчеркнуть, что принцип причинности в приведенном выше виде может быть сформулирован только в РТГ, потому что только в этой теории пространство-время является пространством-временем Минковского и гравитационное поле описывается полем симметричного тензора второго ранга $\phi_{m n}(x)$, где $x^{m}$ - допустимые координаты в пространстве-времени Минковского, $x^{1}, x^{2}, x^{3}$ - пространственноподобные, а $x^{4}$ - времениподобная переменные. 


\section{3. ОДНОРОДНОЕ ГРАВИТАЦИОННОЕ ПОЛЕ В КЛАССИЧЕСКОЙ МЕХАНИКЕ}

В классической механике поле тяготения называется однородным, если его интенсивность постоянна или кусочно-постоянна. Такое поле порождается системой масс, равномерно распределенных на плоскости. Связь между поверхностной плотностью массы $\sigma$ и ускорением $\mathcal{G}$, возникающим под действием поля тяготения, дается соотношением

$$
\mathcal{G}=2 \pi \sigma k>0
$$

где $k$ - гравитационная постоянная Ньютона.

Выбирая оси прямоугольной системы координат $x$ и $y$ в плоскости распределения масс и ось $z$ перпендикулярно к этой плоскости, находим, что движение свободной пробной частицы в этом поле тяготения подчинено уравнениям

$$
\begin{array}{cl}
\frac{d^{2} x}{d t^{2}}=0, & \frac{d^{2} y}{d t^{2}}=0, \\
\frac{d^{2} z}{d t^{2}}+\mathcal{G}=0 \quad \text { для } z>0, & \frac{d^{2} z}{d t^{2}}-\mathcal{G}=0 \quad \text { для } z<0,
\end{array}
$$

где $t$ - ньютоновское время.

В неинерциальной системе отсчета, которая перемешается с постоянным собственным ускорением $\mathcal{G}$, законы движения свободной пробной частищы имеют следуюший Вид:

$$
\frac{d^{2} x}{d t^{2}}=0, \quad \frac{d^{2} y}{d t^{2}}=0, \quad \frac{d^{2} z}{d t^{2}}+\mathcal{G}=0 \quad \text { для любого } z
$$

Здесь $x, y, z$ - координаты частицы в неинерциальной системе.

Несмотря на формальное подобие между системой уравнений $(3.2),(3.3)$ и системой уравнений (3.4), эти два набора законов неэквивалентны. Чтобы заметить это, достаточно сравнить уравнения (3.3) и (3.4). В то время как законы (3.4) могут принять в результате преобразования системы отсчета вид

$$
\frac{d^{2} X}{d t^{2}}=0, \quad \frac{d^{2} Y}{d t^{2}}=0, \quad \frac{d^{2} Z}{d t^{2}}=0 \quad \text { для любого } Z
$$

это невозможно для законов (3.2), (3.3). Неэквивалентность между этими двумя наборами законов отражает тот факт, что в первом случае мы имеем дело с реальным полем тяготения, а во втором присутствует только сила инерции.

Этот пример показьвает, что даже в классической механике есть разница между реальной гравитационной силой, созданной распределением масс, и силой инерции, действующей в неинерциальной системе отсчета. 


\section{4. ОДНОРОДНОЕ ГРАВИТАЦИОННОЕ ПОЛЕ В ОТО}

В своей монографии ([1], гл. 17) Богородский пытался найти ответы на следуюшие вопросы. Сушествует ли однородное гравитационное поле в ОТО? Какая риманова метрика представляет в ОТО гравитационное поле, порожденное бесконечной материальной плоскостью с постоянной поверхностной плотностью массы? Полученные им ответы на эти вопросы приводятся ниже.

Принимая во внимание классические результаты, Богородский ишет решение уравнений Эйнштейна в виде

$$
d s^{2}=-A d x^{2}-A d y^{2}-C d z^{2}+D d t^{2},
$$

где $A, C, D$ - положительные функции, зависящие только от $z$.

Тензор энергии-импульса источников, равномерно распределенных по плоскости $z=0$, имеет вид

$$
T^{m n} \equiv 0 \text { при всех } z \neq 0 .
$$

Для метрики (4.1) и для тензора энергии-импульса (4.2) Богородский делает вывод, что полевые уравнения Эйнштейна выполняются для произвольных функций $A, C, D$, удовлетворяющих следующим уравнениям:

$$
\begin{aligned}
2\left(\frac{A^{\prime}}{A}\right)^{\prime}-\frac{A^{\prime} C^{\prime}}{A C}+\frac{A^{\prime}}{A}\left(\frac{2 A^{\prime}}{A}+\frac{D^{\prime}}{D}\right) & =0, \\
2\left(\frac{D^{\prime}}{D}\right)^{\prime}-\frac{C^{\prime} D^{\prime}}{C D}+\frac{D^{\prime}}{D}\left(\frac{2 A^{\prime}}{A}+\frac{D^{\prime}}{D}\right) & =0, \\
\frac{A^{\prime}}{A}\left(\frac{A^{\prime}}{A}+\frac{2 D^{\prime}}{D}\right) & =0 .
\end{aligned}
$$

Здесь штрих обозначает дифференцирование по координате $z$.

Согласно последнему уравнению имеются две возможности:

$$
A^{\prime}=0 \text { или }\left(\frac{A^{\prime}}{A}+\frac{2 D^{\prime}}{D}\right)=0 .
$$

В первом случае можно положить $A=1$, потому что функции $A, C, D$ определяются с точностью до константы. В этом случае первое уравнение системы (4.3), очевидно, выполняется, и второе уравнение (4.3) принимает вид

$$
\left(\frac{D^{\prime}}{D}\right)^{\prime}-\frac{1}{2} \frac{C^{\prime} D^{\prime}}{C D}+\frac{1}{2}\left(\frac{D^{\prime}}{D}\right)^{2}=0 .
$$

Из (4.5) следует, что $C=a D^{-1} D^{\prime 2}$, где $a$ - вешественная постоянная интегрирования.

Таким образом, в этом случае решение уравнений Эйнштейна имеет вид

$$
A=1, \quad C=a D^{-1} D^{\prime 2},
$$


где $D$ - произвольная функция $z$.

Для второго случая из (4.4) можно взять $A=D^{-2}$ и два первых уравнения (4.3) дают

$$
\left(\frac{D^{\prime}}{D}\right)^{\prime}-\frac{1}{2} \frac{C^{\prime} D^{\prime}}{C D}-\frac{3}{2}\left(\frac{D^{\prime}}{D}\right)^{2}=0 .
$$

Из (4.7) следует $C=b D^{-5} D^{\prime 2}$, где $b$ - постоянная интегрирования.

Таким образом, во втором случае решение уравнений Эйнштейна имеет вид

$$
A=D^{-2}, \quad C=b D^{-5} D^{\prime 2}
$$

где $D$ - произвольная функция $z$.

Далее Богородский находит, что движение свободной пробной частицы в полученном гравитационном поле определяется уравнениями геодезической

$$
\begin{aligned}
& \frac{d^{2} x}{d t^{2}}+\left(\frac{A^{\prime}}{A}-\frac{D^{\prime}}{D}\right) \frac{d x}{d t} \frac{d z}{d t}=0, \quad \frac{d^{2} y}{d t^{2}}+\left(\frac{A^{\prime}}{A}-\frac{D^{\prime}}{D}\right) \frac{d y}{d t} \frac{d z}{d t}=0 \\
& \frac{d^{2} z}{d t^{2}}-\frac{A^{\prime}}{2 C}\left[\left(\frac{d x}{d t}\right)^{2}+\left(\frac{d y}{d t}\right)^{2}\right]+\left(\frac{C^{\prime}}{2 C}-\frac{D^{\prime}}{D}\right)\left(\frac{d z}{d t}\right)^{2}+\frac{D^{\prime}}{2 C}=0
\end{aligned}
$$

Для обсуждаемой выше системы можно видеть, что вертикальное движение со скоростью, равной в начальный момент нулю, описывается системой уравнений

$$
\begin{gathered}
\frac{d^{2} x}{d t^{2}}=0, \quad \frac{d^{2} y}{d t^{2}}=0 \\
\frac{d^{2} z}{d t^{2}}+\left(\frac{C^{\prime}}{2 C}-\frac{D^{\prime}}{D}\right)\left(\frac{d z}{d t}\right)^{2}+\frac{D^{\prime}}{2 C}=0
\end{gathered}
$$

В случае медленного движения член, содержаший скорость, должен быть опушен и уравнение (4.12) принимает вид

$$
\frac{d^{2} z}{d t^{2}}+\frac{D^{\prime}}{2 C}=0
$$

Сравнивая систему уравнений (4.11), (4.13) с классической, Богородский требует выполнения соотношения

$$
\frac{D^{\prime}}{2 C}=\mathcal{G}
$$

Позже мы вернемся к этому условию.

Наконец, принимая константы $a, b$ равными $1 /\left(4 \mathcal{G}^{2}\right)$ и используя соотношение $(4.14)$, из (4.6), (4.8) Богородский получает решения

$$
A=1, \quad C=e^{2 \mathcal{G} z}, \quad D=e^{2 \mathcal{G} z}
$$

и

$$
A=(1-8 \mathcal{G} z)^{\frac{1}{2}}, \quad C=(1-8 \mathcal{G} z)^{-\frac{5}{4}}, \quad D=(1-8 \mathcal{G} z)^{-\frac{1}{4}}
$$


Для первого решения (4.15) тензор кривизны Римана-Кристоффеля тождественно равен нулю. Таким образом, автор заключает, что решение (4.15) не представляет реального гравитационного поля. Легко увидеть с помошью преобразований

$$
X=x, \quad Y=y, \quad Z=\frac{1}{\mathcal{G}}\left[e^{\mathcal{G} z} \operatorname{ch}(\mathcal{G} t)-1\right], \quad T=\frac{1}{\mathcal{G}} e^{\mathcal{G} z} \operatorname{sh}(\mathcal{G} t)
$$

что фундаментальный инвариант (4.1) принимает вид метрики Минковского

$$
d \sigma^{2}=-d X^{2}-d Y^{2}-d Z^{2}+d T^{2}
$$

Свойства и сингулярности неинерциальной системы, характеризуемой соотношениями (4.17), детально описаны в книге [5], гл. 15.

Таким образом, получаем, что первое решение (4.15) соответствует неинерциальной системе отсчета, начало которой движется с постоянным собственным ускорением $\mathcal{G}$ вдоль положительного направления оси Z инерциальной системы. Тензор кривизны Римана-Кристоффеля, соответствующий второму решению (4.16), отличен от нуля. Согласно Богородскому это решение представляет реальное однородное гравитационное поле в ОТО, порожденное рассмотренным распределением масс.

Прежде всего можно заметить, что решение (4.16) имеет сингулярность при $z=$ $1 /(8 \mathcal{G})$, объяснить которую затруднительно.

Вернемся к условию (4.14), наложенному Богородским. В разделе 3 было замечено, что в классической механике движение свободной пробной частицы в реальном гравитационном поле описывается системой уравнений (3.2), (3.3), а не системой (3.4). Таким образом, соотношение (4.14) Богородского должно быть заменено на соотношение

$$
\frac{D^{\prime}}{2 C}= \begin{cases}\mathcal{G}, & z>0 \\ -\mathcal{G}, & z<0\end{cases}
$$

Следовательно, решение Богородского (4.16) следует заменить на

$$
A=(1 \mp 8 \mathcal{G} z)^{\frac{1}{2}}, \quad C=(1 \mp 8 \mathcal{G} z)^{-\frac{5}{4}}, \quad D=(1 \mp 8 \mathcal{G} z)^{-\frac{1}{4}}
$$

где знак плюс соответствует случаю $z<0$, знак минус - случаю $z>0$. Решение (4.20) может быть принято только для $-1 /(8 \mathcal{G})<z<1 /(8 \mathcal{G})$, и сингулярности при $z=$ $\pm 1 /(8 \mathcal{G})$ остаются без какого бы то ни было физического объяснения.

\section{5. ОДНОРОДНОЕ ГРАВИТАЦИОННОЕ ПОЛЕ В РТГ}

Чтобы рассматривать на основе РТГ какую-либо задачу, необходимо решить уравнения $(2.3),(2.4)$ относительно координат исходного пространства-времени Минковского. Физически приемлемым гравитационным полям отвечают только те решения, которые удовлетворяют принципу причинности. Начнем с той же самой отправной точки, 
что и Богородский. Будем искать решение задачи нахождения однородного гравитационного поля в РТГ в виде (4.1). При этом можно использовать два подхода. Их оба мы и рассмотрим.

Сначала воспользуемся уже найденными решениями (4.15), (4.20), удовлетворяющими уравнениям (2.3) (без массовых членов). Проверим, удовлетворяют ли эти решения уравнениям (2.4).

Для решения (4.15) компоненты исходной метрики Минковского и компоненты эффективной римановой метрики совпадают, поскольку это решение появляется при переходе от инерциальной системы отсчета к ускоренной системе отсчета в пространстве-времени Минковского. Таким образом, уравнения (2.4), очевидно, выполняются, $D_{m}$ является оператором ковариантного дифференцирования по отношению к метрике Минковского.

Для решения (4.20), прежде всего, необходимо найти систему отсчета в исходном пространстве-времени Минковского. Эта система отсчета может быть получена в предположении об исчезновении гравитационного поля. При этом для $\sigma=0$ и, следовательно, для $\mathcal{G}=0$ метрика (4.1) в обсужденной выше системе отсчета принимает вид

$$
d \sigma^{2}=-d x^{2}-d y^{2}-d z^{2}+d t^{2} .
$$

Таким образом, для выбранной системы координат компоненты метрической связности $\gamma_{m p}^{n}$ равны нулю и уравнение (2.5) принимает простой вид

$$
\tilde{g}^{m n}, m=0 .
$$

Учитывая соотношения (2.2), (4.1), (4.20), видим, что уравнение (5.2) не выполняется. Таким образом, решение (4.20) не является в РТГ допустимым решением. Для нахождения допустимого в РТГ решения используем процедуру, описанную в книге [3], гл. 13. А именно, будем искать систему координат $\left\{\eta^{i}\right\}=\{X, Y, Z, T\}$, в которой вьполняется уравнение (2.3) (без массовых членов), а уравнение (2.4) устанавливает взаимно однозначное соотношение между наборами координат $\left\{\eta^{i}\right\}$ и $\left\{\xi^{i}\right\}=\{x, y, z, t\}$ в пространстве-времени Минковского. Эта замена делается таким образом, что когда гравитационное поле выключается, то мы оказьваемся в пространстве-времени Минковского с метрикой

$$
d \sigma^{2}=-d X^{2}-d Y^{2}-d Z^{2}+d T^{2} .
$$

Таким образом, компоненты тензора $\gamma_{m p}^{n}$ в этой системе координат тождественно равны нулю. Поскольку компоненты метрики (4.1) зависят только от $z$, перейдем от переменных $\left\{\xi^{i}\right\}$ к переменным $\left\{\eta^{i}\right\}$, предполагая, что

$$
X=x, \quad Y=y, \quad Z=Z(z), \quad T=t .
$$

Запишем уравнения (2.4) в выбранной системе координат в форме (см. также соотношения (13.17), (13.22) из [3])

$$
\frac{\partial}{\partial \xi^{m}}\left(\sqrt{-g(\xi)} g^{m n}(\xi) \frac{\partial \eta^{p}}{\partial \xi^{n}}\right)=0 .
$$


Для преобразований (5.4) с учетом соотношений (4.1), (4.20) уравнение (5.5) принимает вид

$$
\frac{d}{d z}\left((1 \mp 8 \mathcal{G} z) \frac{d Z}{d z}\right)=0 .
$$

Интегрируя это уравнение и выбирая константу интегрирования так, что для $\mathcal{G}$, стремяшейся к нулю, $Z$ стремится к $z$, получаем

$$
Z=\mp \frac{1}{8 \mathcal{G}} \ln (1 \mp 8 \mathcal{G} z) .
$$

Чтобы найти компоненты метрики (4.1) в системе координат (5.4), (5.7), достаточно воспользоваться тензорным законом преобразования,

$$
\begin{array}{llll}
A=e^{-4 \mathcal{G} Z}, & C=e^{-6 \mathcal{G} Z}, & D=e^{2 \mathcal{G} Z} & \text { при } Z>0, \\
A=e^{4 \mathcal{G} Z}, & C=e^{6 \mathcal{G} Z}, & D=e^{-2 \mathcal{G} Z} & \text { при } Z<0 .
\end{array}
$$

Решение (5.8) удовлетворяет полной системе уравнений (2.3) (без массовых членов), (2.4). Это решение регулярно при любом $Z \neq 0$. Тем не менее оно не является дифференцируемым при $Z=0$, и при переходе через эту плоскость производные функций $A, C, D$ имеют конечные скачки. Конечно, появление этой сингулярности, сосредоточенной на плоскости $Z=0$, отражает тот факт, что это реальное гравитационное поле имеет своим источником систему масс, распределенную по соответствующей плоскости. Мы видим также, что условие (4.19) может быть вьполнено только приближенно, поскольку, например, из (5.8) следует, что

$$
\frac{D^{\prime}}{2 C}=\mathcal{G} e^{8 \mathcal{G} Z} \quad \text { для любого } Z>0 .
$$

В то же время мы замечаем, что это приближение достаточно корректно. В самом деле, если использовать обычные единицы, то

$$
\frac{D^{\prime}}{2 C}=\frac{\mathcal{G}}{c^{2}} e^{\frac{8 \mathcal{G} Z}{c^{2}}} \quad \text { для любого } Z>0,
$$

где $c$ - скорость света в вакууме, соответствующая инерциальной системе. Следовательно, для любого положительного $Z$ при условии

$$
Z \ll \frac{c^{2}}{\mathcal{G}}
$$

отношение $D^{\prime} / 2 C$ может считаться приблизительно постоянным.

Анализ в РТГ не может быть на этом завершен, так как полученные решения должны также быть проверены принципом причинности. Для первого решения (4.15) принцип причинности, очевидно, выполняется, поскольку в этом случае, как уже говорилось, метрика Минковского и риманова метрика совпадают. Таким образом, решение (4.15) является допустимым решением и в РТГ. Его физический смысл уже был прояснен. 
Для второго решения (5.8) с учетом вида (5.3) исходного пространства-времени Минковского вектор $u=(1,0,0,1)$ является изотропным вектором пространства Минковского. Следовательно, условие (2.7) выполняется, если

$$
e^{2 \mathcal{G} Z} \leqslant e^{-4 \mathcal{G} Z} \text { для } Z>0 \text { и } e^{-2 \mathcal{G} Z} \leqslant e^{4 \mathcal{G} Z} \text { для } Z<0 .
$$

Эти условия не выполняются ни при каких $Z>0$ или $Z<0$, если ускорение $\mathcal{G}$ не равно нулю, и мы заключаем, что согласно РТГ не может существовать обобщения однородного гравитационного поля в смысле Богородского.

Можно также использовать для нахождения решения обсуждаемой проблемы в РТГ другой подход. Из уравнения (2.3) (без массовых членов) при $T_{n}^{m} \equiv 0$ для $z \neq 0$ мы получаем решения (4.6), (4.8). Подчеркнем, что в (4.6), (4.8) функция $D(z)$ произвольна. Это ясно показывает, что эйнштейновские полевые уравнения недостаточны для нахождения единственного гравитационного поля, порожденного рассмотренным распределением масс. Определим неизвестную функцию $D(z)$, используя уравнения (2.4).

Рассмотрим галилеевы координаты $x, y, z, t$ инерциальной системы. Тогда уравнения (2.4) принимают простую форму (5.2). Принимая во внимание (2.2), (4.1), (4.6), (4.8), получаем из (5.2)

$$
D(z)=p e^{q z}
$$

где $p$ и $q$-вешественные постоянные. Таким образом, подставляя (5.13) в (4.6), получаем согласно РТГ первое решение

$$
A=1, \quad C=a p e^{q z}, \quad D=p e^{q z} .
$$

Из (4.8) находим второе решение

$$
A=p^{-2} e^{-2 q z}, \quad C=b p^{-3} e^{-3 q z}, \quad D=p e^{q z} .
$$

Для решения (5.14) тензор кривизны Римана-Кристоффеля тождественно равен нулю, а для решения (5.15) он отличен от нуля.

Константы $a, b, p, q$ должны определяться из принципа соответствия: после выключения гравитационного поля кривизна пространства исчезает и мы оказываемся в пространстве-времени Минковского в выбранной системе отсчета. Таким образом, в выбранной системе отсчета уравнения движения становятся классическими. Из принципа геометризации РТГ уравнения движения в рассматриваемом гравитационном поле даются уравнениями (4.9), (4.10). В случае медленного вертикального движения эта система уравнений переходит в систему $(4.11),(4.13)$. Таким образом, из принципа соответствия мы должны получать соотношение (4.14) для решения (5.14) и соотношение (4.19) для решения (5.15).

Согласно этому же принципу метрика должна стремиться к галилеевой метрике при $\mathcal{G} \rightarrow 0$. Таким образом, в первом случае мы получаем решение (4.15), а во втором случае - решение (5.8). Для последнего решения соотношение (4.19) выполняется только приближенно, о чем мы уже говорили. 
Итак, на основе двух подходов мы получили один и тот же результат: решение (4.15) представляет собой силы инерции, а решение (5.8) не может быть принято в качестве реального гравитационного поля, созданного рассматриваемым распределением масс, поскольку оно не удовлетворяет принципу причинности (см. (5.12)).

\section{6. ВАЖНОСТЬ ПРИНЦИПА ПРИЧИННОСТИ В РТГ}

Пример, который будет рассмотрен в этом разделе, ясно указывает на важность принципа причинности для вывода о неприемлемости решения (5.8) в РТГ. Будет показано, что в найденном пространстве-времени существуют свободные пробные частицы, которые движутся быстрее, чем свет в вакууме.

Возьмем свободную пробную частицу, расположенную в начальный момент времени $t=0$ на расстоянии $h>0$ от плоскости $z=0$, где сконцентрирована масса. Для простоты рассмотрим задачу в плоскости $x O z$. В момент $t=0$, когда частица занимает указанную позицию

$$
\begin{aligned}
& x(0)=0, \\
& z(0)=h>0,
\end{aligned}
$$

ее отпускают. Из принципа геометризации следует, что если мы хотим изучать поведение этой частицы под влиянием рассматриваемого однородного гравитационного поля, мы можем изучить ее поведение в следуюшем эффективном римановом пространстве-времени (см. (5.8)):

$$
d s^{2}=-e^{-4 \mathcal{G} z} d x^{2}-e^{-6 \mathcal{G} z} d z^{2}+e^{2 \mathcal{G} z} d t^{2} .
$$

Принимая во внимание уравнения геодезических $(4.9),(4.10)$ и выражение для римановой метрики (6.3), видим, что траектория частицы описывается уравнениями

$$
\begin{gathered}
\frac{d^{2} x}{d t^{2}}-6 \mathcal{G} \frac{d x}{d t} \frac{d z}{d t}=0 \\
\frac{d^{2} z}{d t^{2}}+2 \mathcal{G} e^{2 \mathcal{G} z}\left(\frac{d x}{d t}\right)^{2}-5 \mathcal{G}\left(\frac{d z}{d t}\right)^{2}+\mathcal{G} e^{8 \mathcal{G} z}=0
\end{gathered}
$$

Мы также считаем, что в начальный момент $t=0$

$$
\begin{aligned}
& \dot{x}(0)=a>0, \\
& \dot{z}(0)=b>0,
\end{aligned}
$$

где $a, b$ - вешественные постоянные.

Решая уравнение (6.4) и принимая во внимание соотношения (6.2), (6.6), получаем

$$
\dot{x}(t)=a e^{6 \mathcal{G}(z-h)} .
$$


Решая также уравнение (6.5), с учетом (6.2), (6.6), (6.7) получаем

$$
\dot{z}(t)=e^{4 \mathcal{G} z} \sqrt{1+L e^{2 \mathcal{G} z}-a^{2} e^{-12 \mathcal{G} h} e^{6 \mathcal{G} z}} .
$$

Здесь вещественная постоянная

$$
L=b^{2} e^{-10 \mathcal{G} h}+a^{2} e^{-8 \mathcal{G} h}-e^{-2 \mathcal{G} h} .
$$

Теперь выделим в (6.3) времениподобную и пространственноподобную части,

$$
d s^{2}=d \sigma^{2}-d l^{2}
$$

где

$$
\begin{aligned}
d \sigma^{2} & =e^{2 \mathcal{G} z} d t^{2}, \\
d l^{2} & =e^{-4 \mathcal{G} z} d x^{2}+e^{-6 \mathcal{G} z} d z^{2} .
\end{aligned}
$$

Таким образом, если одно локальное событие имеет координаты $(x, 0, z, t)$, а другое $(x+d x, 0, z+d z, t+d t)$, то наблюдатель в точке $(x, 0, z, t)$ с 4-скоростью $(d x / d s, 0, d z / d s$, $d t / d s)$ измеряет пространственный интервал $d l$ и интервал собственного времени $d \sigma$ меж ду двумя событиями.

Скорость частицы в рассматриваемом эффективном римановом пространстве-времени $v\left(v^{1}=d x / d t, 0, v^{3}=d z / d t\right)$ имеет следующую абсолютную величину:

$$
v^{2}=\frac{d l^{2}}{d t^{2}}=e^{-4 \mathcal{G} z} \dot{x}^{2}+e^{-6 \mathcal{G} z} \dot{z}^{2}
$$

Естественно потребовать, чтобы начальная скорость нашей частицы была меньше скорости света в вакууме. Таким образом, из (6.2), (6.6), (6.7), (6.14) получаем неравенство

$$
e^{-4 \mathcal{G} h} a^{2}+e^{-6 \mathcal{G} h} b^{2}<1 .
$$

Принимая во внимание (6.10), (6.15), находим следуюшее ограничение на константу $L$ :

$$
L<e^{-4 \mathcal{G} h}-e^{-2 \mathcal{G} h} .
$$

Мы считали, что $h>0$, поэтому из (6.16) следует

$$
L<0
$$

Теперь покажем, что для выбранного случая имеются некоторые координаты $z$, в которых скорость частицы будет превосходить скорость света в вакууме, т.е. найдется такое значение $z$, что

$$
v^{2}(z)>1
$$


Подставляя (6.8), (6.9) в (6.14), видим, что неравенство (6.18) эквивалентно следуюшемy:

$$
e^{2 \mathcal{G} z}+L e^{4 \mathcal{G} z}>1 \text {. }
$$

Принимая во внимание (6.17), можно получить, что при

$$
-\frac{1}{4}<L<0
$$

т.е. при некоторых условиях, наложенных на начальные значения скорости, для любого $z$, удовлетворяющего неравенству

$$
\frac{1}{2 \mathcal{G}} \ln \left(\frac{-1+\sqrt{1+4 L}}{2 L}\right)<z<\frac{1}{2 \mathcal{G}} \ln \left(\frac{-1-\sqrt{1+4 L}}{2 L}\right),
$$

выполняется неравенство (6.19). Например, ограничения (6.20) выполняются для следуюших $a$ и $b$ :

$$
\begin{aligned}
& a=\rho e^{2 \mathcal{G} h} \cos \theta, \\
& b=\rho e^{3 \mathcal{G} h} \sin \theta \text { при } \theta \in\left(0, \frac{\pi}{2}\right), 0<\rho<1, \quad \rho^{2}>1-\frac{\left(e^{2 \mathcal{G} h}-2\right)^{2}}{4} .
\end{aligned}
$$

Следовательно, если мы рассмотрим в эффективном римановом пространстве-времени (5.8) свободную пробную частицу, которая занимает в начальный момент времени положение (6.1), (6.2) и имеет скорость (6.6), (6.7), так что, принимая во внимание (6.10), вещественные константы $a, b$ удовлетворяют ограничениям (6.20), то окажется, что эта частица движется быстрее, чем свет в вакууме.

\section{7. ЗАКЛЮЧЕНИЕ}

Как мы видели в классической механике, ОТО и РТГ, если система отсчета движется с постоянным собственным ускорением относительно инерциальной системы, то поле инерции, созданное силой инерции, является постоянным полем. Выражения для элемента длины Минковского (4.15) в ОТО и РТГ совпадают. В классической механике было сказано, что это постоянное поле неотличимо от однородного гравитационного поля, созданного бесконечной материальной плоскостью. Но из (3.2), (3.3) и (3.4) мы видели, что между двумя полями имеется различие, даже в классической механике. Это происходит потому, что гравитационная сила является силой притяжения. В ОТО и РТГ различие между постоянным полем, созданным силой инерции, и однородным гравитационным полем, вызванным присутствием масс, является сушественным. Решение Богородского для однородного гравитационного поля в ОТО дается формулами (4.16). Как мы уже говорили, это решение содержит неустранимую сингулярность. Решение, найденное в РТГ, имеет вид (5.8). К сожалению, это решение не удовлетворяет принципу 
причинности в РТГ и должно быть отклонено. Действительно, в полученном пространстве-времени (5.8) скорость свободной пробной частицы может превышать скорость света в вакууме. Заканчивая проведенный анализ, можно сделать вывод, что проблема нахождения в РТГ гравитационного поля, порожденного равномерным распределением масс на бесконечной плоскости, является интересной и по-прежнему остается открыmoй.

Благодарности. Мне хочется поблагодарить моего учителя Е. Сооса за многочисленные полезные и интересные обсуждения, стимулировавшие меня при выполнении этой работы. Мне бы хотелось также поблагодарить В. Соловьева за удачный перевод этой статьи на русский язык.

\section{Список литературы}

[1] А. Ф. Богородский. Всемирное тяготение. Киев: Наукова думка, 1971.

[2] D. Ionescu, E. Soós. Consequences of the Causality Principle in the Relativistic Theory of Gravitation. In: Proceedings of the XXIII International Workshop on High Energy Physics and Field Theory (to be published), Protvino (Russia), June 21-23, 2000.

[3] A. A. Logunov, M. Mestvirishvili. The Relativistic Theory of Gravitation. M.: Mir, 1989.

[4] A. А. Логунов. Релятивистская теория гравитации и принцип Маха. Дубна, 1996.

[5] А. И. Жуков. Введение в теорию относительности. М.: Физматгиз, 1961.

Поступила в редакцию 5.V.2001 г. 